\title{
Effects of Anti-Malarial Drug, Hydroxychloroquine, on Glucose and Lipid Metabolism in Japanese Population
}

\author{
Yoshinori Masui ${ }^{a}$, Hidekatsu Yanai ${ }^{b, c}$, Kenichi Hiraga ${ }^{a}$, \\ Naonori Tsuda ${ }^{\text {a }}$, Toshikazu Kano ${ }^{\mathrm{a}}$
}

\begin{abstract}
Background: Hydroxychloroquine (HCQ) is a synthetic anti-malarial drug and has been used for the treatment of rheumatic diseases. Recently, HCQ has been reported to improve lipid and glucose metabolism in patients with rheumatic diseases. However, effects of HCQ on lipid and glucose metabolism in Japanese remain unknown.

Methods: We picked up patients who had been prescribed HCQ for 1 month or longer between September 2015 and August 2018. We compared the data at baseline and at $1-11$ months after the start of HCQ. We excluded patients who discontinued to take HCQ due to adverse reaction, and patients who had taken daily more than $20 \mathrm{mg}$ of prednisolone with HCQ.
\end{abstract}

Results: We found 40 patients, and excluded nine patients who discontinued to take HCQ due to adverse reactions and five patients who had taken daily more than $20 \mathrm{mg}$ of prednisolone in addition to HCQ, and we analyzed 26 patients. Twenty-two systemic lupus erythematosus (SLE) patients and 4 rheumatoid arthritis (RA) patients had been prescribed HCQ. Hemoglobin A1c (HbAlc) showed a significant decrease and tendency to decrease at 5 and 6 months after the start of HCQ, respectively. At other time points, HbA1c showed non-significant decrease. Serum triglyceride showed a significant decrease at 1, 3, 7, 8 and 11 months after the start of HCQ. At other time points, serum triglyceride showed non-significant decrease. Serum high-density lipoprotein-cholesterol (HDL-C) did not show any significant changes at every time point. Serum low-density lipoproteincholesterol (LDL-C) showed a significant decrease at 2 months after the start of HCQ and showed tendency to decrease after 7 and 10 months. At other time points, serum LDL-C showed non-significant

Manuscript submitted September 17, 2019, accepted October 1, 2019

aDepartment of Rheumatism and Collagen Diseases, Kohnodai Hospital, National Center for Global Health and Medicine, Chiba, Japan

bDepartment of Diabetes, Endocrinology and Metabolism, National Center for Global Health and Medicine Kohnodai Hospital, Chiba, Japan

${ }^{\mathrm{c}}$ Corresponding Author: Hidekatsu Yanai, Department of Diabetes, Endocrinology and Metabolism, National Center for Global Health and Medicine Kohnodai Hospital, 1-7-1 Kohnodai, Ichikawa, Chiba 272-8516, Japan. Email: dyanai@hospk.ncgm.go.jp

doi: https://doi.org/10.14740/jem611 decrease. Serum non-HDL-C showed a significant decrease at 2, 3 and 7 months after the start of HCQ and showed tendency to decrease after 1 and 5 months. At other time points, serum non-HDL-C showed non-significant decrease.

Conclusion: HCQ was associated with reduction of $\mathrm{HbA} 1 \mathrm{c}$, serum triglyceride, LDL-C and non-HDL-C levels in SLE and RA patients.

Keywords: Diabetes; Hydroxychloroquine; Serum lipids; Systemic lupus erythematosus

\section{Introduction}

Hydroxychloroquine (HCQ) is a synthetic anti-malarial drug and has been used for the treatment of rheumatic diseases such as systemic lupus erythematosus (SLE) and rheumatoid arthritis (RA). HCQ has been proved to play its role in immune regulation through a variety of mechanisms, including inhibition of autophagy, antigen presentation and cytokine chemotaxis. HCQ also reduces the production of pro-inflammatory cytokines, inhibits matrix metalloproteinases, blocks T- and B-cell receptors and participates in toll-like receptor signaling $[1,2]$. Recently, HCQ has been reported to improve lipid and glucose metabolism, which may help reduce the high cardiovascular risk in SLE patients [3]. However, effects of HCQ on lipid and glucose metabolism in Japanese remain unknown. Therefore, we retrospectively studied the changes in serum lipid and hemoglobin A1c (HbA1c) levels after the start of HCQ in SLE and RA patients who had been prescribed HCQ.

\section{Materials and Methods}

This study was approval by the Institutional Ethics Committee in National Center for Global Health and Medicine (NCGMG-003045), and was also performed in accordance with the Declaration of Helsinki.

The recruitment of studied subjects was shown in Figure 1. Briefly, we picked up patients who had been prescribed HCQ for 1 month or longer between September 2015 and August 2018 based on medical charts. We compared the data at 


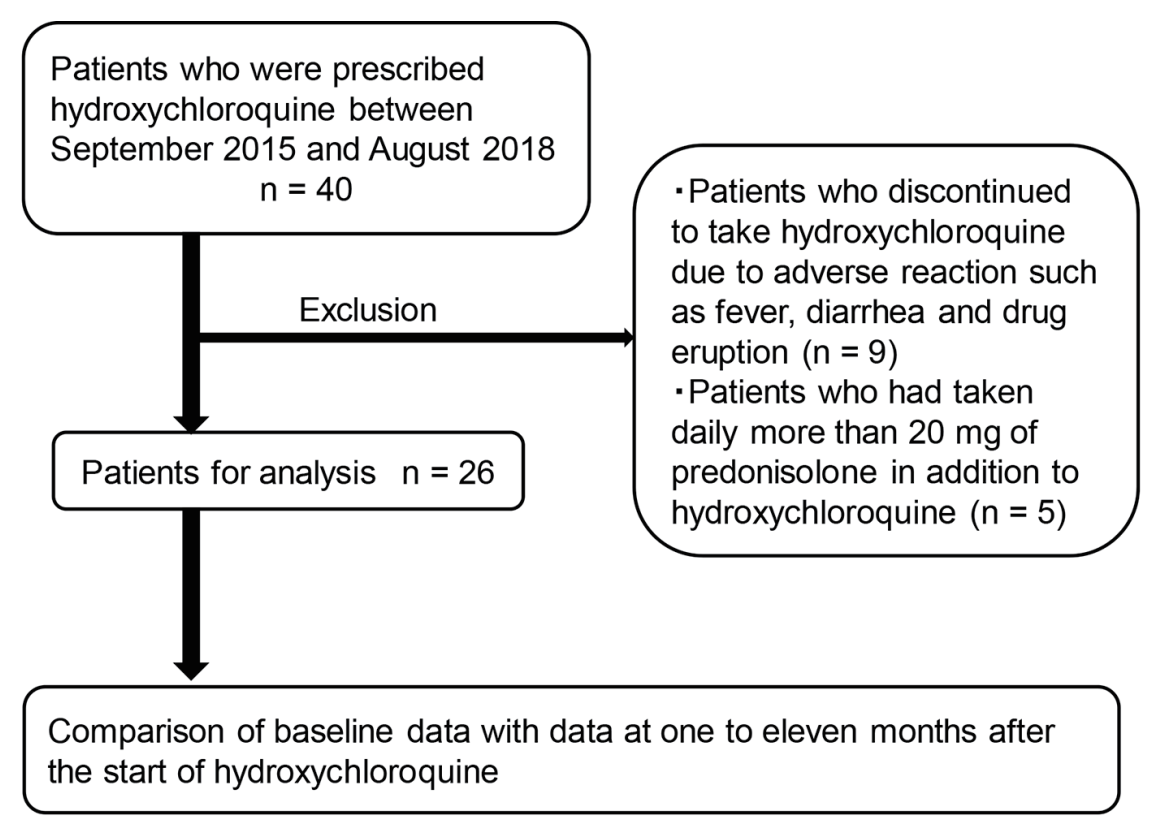

Figure 1. The recruitment of studied subjects.

baseline and at 1 - 11 months after the start of HCQ. We excluded patients who discontinued to take HCQ due to adverse reactions such as fever, diarrhea and drug eruption, and also excluded patients who had taken daily more than $20 \mathrm{mg}$ of prednisolone in addition to HCQ.

Comparison of the variables determined before and after was analyzed by a paired Student's $t$-test. All data are expressed as mean \pm standard deviation $(\mathrm{SD}) . \mathrm{P}<0.05$ and $\mathrm{P}<$ 0.1 were considered to be statistically significant and to show tendency, respectively.

\section{Results}

We found 40 patients who had been prescribed HCQ for 1 month or longer between September 2015 and August 2018, and excluded nine patients who discontinued to take HCQ due to adverse reactions and five patients who had taken daily more than $20 \mathrm{mg}$ of prednisolone in addition to HCQ (Fig. 1). Therefore, we analyzed 26 patients. Clinical data of studied subjects were shown in Table 1. Twenty-two SLE patients and four RA patients had been prescribed HCQ. Three patients with diabetes and seven patients with dyslipidemia were included in this study. Daily dose of HCQ and other medication at baseline were shown in Table 2. Twenty-two patients had taken prednisolone, and mean daily dose was $6.5 \mathrm{mg}$. Two diabetic patients had taken oral anti-diabetic drugs, and six and two dyslipidemia patients had taken statin and fibrate, respectively. During the study period, decrease of daily dose of anti-diabetic drugs, statin and fibrate were observed; however, increase of daily dose

Table 1. Clinical Data at Baseline $(n=26)$

\begin{tabular}{|c|c|}
\hline \multicolumn{2}{|l|}{ Anthropometric data } \\
\hline Sex (female, n (\%)) & $22(84.6 \%)$ \\
\hline Age (years, mean $\pm \mathrm{SD})$ & $46 \pm 16$ \\
\hline Body weight $(\mathrm{kg}$, mean $\pm \mathrm{SD})$ & $51.8 \pm 4.6$ \\
\hline Body mass index $\left(\mathrm{kg} / \mathrm{m}^{2}\right.$, mean $\left.\pm \mathrm{SD}\right)$ & $20.8 \pm 2.4$ \\
\hline Systemic lupus erythematosus (n (\%)) & $22(84.6 \%)$ \\
\hline Rheumatoid arthritis (n (\%)) & $4(15.4 \%)$ \\
\hline \multicolumn{2}{|l|}{ Metabolic diseases } \\
\hline Diabetes (n (\%)) & $3(11.5 \%)$ \\
\hline Dyslipidemia (n (\%)) & $7(26.9 \%)$ \\
\hline
\end{tabular}

SD: standard deviation. 
Table 2. Daily Dose of Hydroxychloroquine and Other Medication at Baseline

\begin{tabular}{|c|c|}
\hline Daily dose of hydroxychloroquine $(\mathrm{mg}$, mean $\pm \mathrm{SD})$ & $284.6 \pm 67.5$ \\
\hline \multicolumn{2}{|l|}{ For rheumatic diseases } \\
\hline \multicolumn{2}{|l|}{ Prednisolone } \\
\hline Daily dose (mg) & $6.5 \pm 3.5$ \\
\hline Immunosuppressants (n (\%)) & $7(26.9 \%)$ \\
\hline Insulin (n (\%)) & $0(0 \%)$ \\
\hline \multicolumn{2}{|l|}{ For dyslipidemia } \\
\hline Statin $(\mathrm{n}(\%))$ & $6(23.1 \%)$ \\
\hline Fibrate $(\mathrm{n}(\%))$ & $2(7.7 \%)$ \\
\hline
\end{tabular}

SD: standard deviation.

and additional administration of such drugs were not observed.

Changes in HbAlc were shown in Table 3. HbAlc showed a significant decrease and tendency to decrease at 5 and 6 months after the start of $\mathrm{HCQ}$, respectively. At other time points, HbA1c showed non-significant decrease.

Changes in serum triglyceride were shown in Table 4. Serum triglyceride showed a significant decrease at 1, 3, 7, 8 and 11 months after the start of HCQ. At other time points, serum triglyceride showed non-significant decrease. Changes in serum high-density lipoprotein-cholesterol (HDL-C) were shown in Table 5. Serum HDL-C did not show any significant changes at every time point.

Changes in serum low-density lipoprotein-cholesterol (LDL-C) were shown in Table 6. Serum LDL-C showed a significant decrease at 2 months after the start of HCQ and showed tendency to decrease after 7 and 10 months. At other time points, serum LDL-C showed non-significant decrease. Changes in serum non-HDL-C were shown in Table 7. Serum non-HDL-C showed a significant decrease at 2, 3 and 7 months after the start of HCQ and showed tendency to decrease after 1 and 5 months. At other time points, serum non-HDL-C showed non-significant decrease.

\section{Discussion}

The metabolic syndrome (MetS) is now recognized as a chronic proinflammatory state that aggravates insulin resistance. MetS and atherosclerosis are more prevalent in patients with SLE and RA [4]. There is an urgent need for clinical trials to examine both the lipid-lowering and inflammatory hypotheses of atherosclerosis in SLE and RA. Novel targeted therapies in development may also have a major impact on future coronary heart disease risk in rheumatic diseases. Dysregulation of cytokines and adipokines is a common feature in both SLE and MetS, suggesting a complex relationship among autoimmunity, obesity, inflammation and atherosclerosis [5]. While lifestyle modifications and targeting dyslipidemia, hyperten-

Table 3. Change of $\mathrm{HbA} 1 \mathrm{c}$ After the Start of Hydroxychloroquine

\begin{tabular}{lllll}
\hline Month & $\mathbf{n}$ & Values at baseline & Values after hydroxychloroquine use & P value \\
\hline 1 & 11 & $5.91 \pm 0.60$ & $5.87 \pm 0.54$ & 0.397 \\
2 & 11 & $5.83 \pm 0.59$ & $5.75 \pm 0.48$ & 0.221 \\
3 & 10 & $6.02 \pm 0.50$ & $5.92 \pm 0.44$ & 0.348 \\
4 & 11 & $5.88 \pm 0.62$ & $5.74 \pm 0.55$ & 0.202 \\
5 & 7 & $5.99 \pm 0.57$ & $5.74 \pm 0.41$ & 0.075 \\
6 & 9 & $5.91 \pm 0.69$ & $5.66 \pm 0.47$ & 0.048 \\
7 & 8 & $6.01 \pm 0.55$ & $5.76 \pm 0.32$ & 0.154 \\
8 & 7 & $5.73 \pm 0.72$ & $5.50 \pm 0.51$ & 0.331 \\
9 & 7 & $5.86 \pm 0.77$ & $5.71 \pm 0.50$ & 0.441 \\
11 & 6 & $5.80 \pm 0.76$ & $5.52 \pm 0.50$ & 0.164 \\
\hline
\end{tabular}

HbA1c: hemoglobin A1c. 
Table 4. Change of Serum Triglyceride ( $\mathrm{mg} / \mathrm{dL}$ ) After the Start of Hydroxychloroquine

\begin{tabular}{lllll}
\hline Month & $\mathbf{n}$ & Values at baseline & Values after hydroxychloroquine use & P value \\
\hline 1 & 12 & $101.0 \pm 27.1$ & $93.7 \pm 26.8$ & 0.005 \\
2 & 10 & $102.3 \pm 27.9$ & $99.3 \pm 30.5$ & 0.657 \\
3 & 11 & $102.6 \pm 21.7$ & $90.1 \pm 24.5$ & 0.011 \\
4 & 13 & $99.2 \pm 25.7$ & $96.2 \pm 32.5$ & 0.618 \\
5 & 10 & $101.8 \pm 19.7$ & $96.5 \pm 16.2$ & 0.228 \\
6 & 9 & $88.1 \pm 20.7$ & $81.7 \pm 20.6$ & 0.318 \\
7 & 9 & $105.2 \pm 22.8$ & $88.8 \pm 17.6$ & 0.006 \\
8 & 8 & $98.0 \pm 28.7$ & $81.5 \pm 24.1$ & 0.045 \\
9 & 9 & $93.2 \pm 25.2$ & $88.0 \pm 18.8$ & 0.438 \\
11 & 9 & $97.2 \pm 27.0$ & $95.0 \pm 30.5$ & 0.876 \\
\hline
\end{tabular}

Table 5. Change of Serum HDL-C (mg/dL) After the Start of Hydroxychloroquine

\begin{tabular}{lllll}
\hline Month & $\mathbf{n}$ & Values at baseline & Values after hydroxychloroquine use & P value \\
\hline 1 & 13 & $59.5 \pm 22.9$ & $59.1 \pm 21.5$ & 0.722 \\
2 & 11 & $59.2 \pm 24.5$ & $57.8 \pm 24.4$ & 0.393 \\
3 & 12 & $61.2 \pm 23.2$ & $61.0 \pm 23.0$ & 0.908 \\
\hline 4 & 14 & $58.2 \pm 21.3$ & $60.4 \pm 21.7$ & 0.104 \\
5 & 10 & $60.7 \pm 23.6$ & $63.3 \pm 21.8$ & 0.164 \\
6 & 10 & $61.6 \pm 22.4$ & $59.1 \pm 24.4$ & 0.231 \\
7 & 10 & $66.4 \pm 21.8$ & $64.9 \pm 20.9$ & 0.696 \\
8 & 8 & $62.9 \pm 24.5$ & $62.8 \pm 22.9$ & 0.955 \\
9 & 10 & $63.2 \pm 22.9$ & $67.5 \pm 21.6$ & 0.217 \\
11 & 10 & $61.8 \pm 22.5$ & $65.4 \pm 22.9$ & 0.287 \\
\hline
\end{tabular}

HDL-C: high-density lipoprotein-cholesterol.

Table 6. Change of Serum LDL-C (mg/dL) After the Start of Hydroxychloroquine

\begin{tabular}{lllll} 
Month & $\mathbf{n}$ & Values at baseline & Values after hydroxychloroquine use & P value \\
\hline 1 & 14 & $157.4 \pm 102.6$ & $141.5 \pm 138.0$ & 0.382 \\
2 & 13 & $179.9 \pm 100.3$ & $134.8 \pm 68.1$ & 0.009 \\
3 & 13 & $143.2 \pm 64.6$ & $131.7 \pm 58.1$ & 0.373 \\
4 & 16 & $173.9 \pm 101.5$ & $149.6 \pm 128.1$ & 0.133 \\
5 & 10 & $151.0 \pm 75.2$ & $115.9 \pm 47.2$ & 0.132 \\
6 & 12 & $190.8 \pm 106.5$ & $176.8 \pm 127.1$ & 0.551 \\
7 & 11 & $133.1 \pm 65.9$ & $101.5 \pm 37.3$ & 0.099 \\
8 & 9 & $182.9 \pm 132.5$ & $158.1 \pm 125.1$ & 0.291 \\
9 & 12 & $180.8 \pm 108.7$ & $155.6 \pm 95.5$ & 0.558 \\
11 & 11 & $180.3 \pm 120.3$ & $134.0 \pm 52.3$ & 0.098 \\
\hline
\end{tabular}

LDL-C: low-density lipoprotein-cholesterol. 
Table 7. Change of Serum Non-HDL-C (mg/dL) After the Start of Hydroxychloroquine

\begin{tabular}{lllll}
\hline Month & $\mathbf{n}$ & Values at baseline & Values after hydroxychloroquine use & P value \\
\hline 1 & 13 & $135.2 \pm 26.9$ & $120.8 \pm 20.2$ & 0.051 \\
2 & 11 & $143.3 \pm 22.8$ & $127.8 \pm 27.4$ & 0.038 \\
3 & 12 & $132.7 \pm 28.3$ & $119.0 \pm 23.9$ & 0.008 \\
4 & 14 & $133.7 \pm 26.0$ & $126.5 \pm 24.3$ & 0.157 \\
5 & 9 & $131.4 \pm 25.9$ & $119.8 \pm 17.6$ & 0.074 \\
6 & 10 & $127.8 \pm 24.2$ & $122.4 \pm 22.2$ & 0.579 \\
7 & 10 & $134.0 \pm 29.1$ & $111.7 \pm 15.1$ & 0.011 \\
8 & 8 & $129.5 \pm 26.2$ & $115.9 \pm 18.4$ & 0.202 \\
9 & 10 & $131.9 \pm 26.6$ & $122.7 \pm 19.2$ & 0.278 \\
10 & 10 & $133.2 \pm 24.9$ & $124.5 \pm 25.7$ & 0.361 \\
\hline
\end{tabular}

HDL-C: high-density lipoprotein-cholesterol.

sion and diabetes is essential, there is little information on the efficacy and safety of HCQ in alleviating insulin resistance in SLE [5]. We observed a significant decrease of HbA1c at 6 months after the start of HCQ and decrease of HbAlc at every time point. HCQ has been reported to play a role in improving obesity-induced lipotoxicity and insulin resistance though the peroxisome proliferator-activated receptor gamma pathway in obese mice [6]. A systematic review and meta-analysis which studied metabolic and cardiovascular benefits of HCQ in patients with RA showed that diabetes incidence was lower for HCQ ever users than never users (hazard ratio (HR) 0.59 (95\% confidence interval (CI) 0.49 to 0.70 )) [7]. HCQ may improve glucose metabolism due to amelioration of insulin resistance by reduced inflammatory state.

The meta-analysis using RA patients showed that the mean differences (MDs) between HCQ users and non-users in levels of total cholesterol (TC), LDL-C, HDL-C and triglyceride were $-9.8(95 \%$ CI -14.0 to -5.6$),-10.6$ (95\% CI -14.2 to -7.0$),+4.1(95 \%$ CI 2.2 to 6.0$)$ and -19.2 (95\% CI -27.2 to -11.1), respectively [7]. Although serum HDL-C did not show any significant changes at every time point in present study, serum triglyceride, LDL-C and non-HDL-C showed a significant decrease at several time points and showed a decrease at every time point after the start of HCQ as compared with baseline. In a systematic review and meta-analysis which studied effects of chloroquine (CQ) or HCQ on serum lipids in SLE patients, compared with the control group, TC, triglyceride, LDL-C, very low-density lipoprotein (VLDL)-C were associated with a significant decrease, respectively (weighted the mean difference $(\mathrm{WMD})=-21.40 \mathrm{mg} / \mathrm{dL}, 95 \% \mathrm{CI}-27.62$ to $-15.18, \mathrm{P}<$ $0.00001),(\mathrm{WMD}=-29.07 \mathrm{mg} / \mathrm{dL}, 95 \% \mathrm{CI}-45.28$ to $-12.86, \mathrm{P}$ $=0.0004),(\mathrm{WMD}=-16.25 \mathrm{mg} / \mathrm{dL}, 95 \% \mathrm{CI}-28.82$ to $-3.68, \mathrm{P}=$ $0.01),(\mathrm{WMD}=-6.41 \mathrm{mg} / \mathrm{dL}, 95 \% \mathrm{CI}-12.39$ to $0.44, \mathrm{P}=0.04)$; however, the change of HDL-C did not reach statistically significance $(\mathrm{WMD}=4.42 \mathrm{mg} / \mathrm{dL}, 95 \% \mathrm{CI}-1.21$ to $10.06, \mathrm{P}=$ 0.12 ), which completely agreed with our results [8].

The mechanism of HCQ or CQ decreasing the serum lipid among SLE patients remains unclear. Chen et al reported that CQ treatment of rat cells in culture results in the increase of hydroxy-methyl-glutaryl coenzyme A (HMG-CoA) reductase activity [9], which was a rate-limiting enzyme in the process of cholesterol synthetize in hepatocytes. After CQ administration, the hepatic activities of lysosomal enzymes were increased and cholesterol saturation of bile decreased by $22 \%$ in rat [10]. Sachet et al reported that CQ can up-regulate LDLreceptor in SLE [11].

Insulin resistance induced by inflammation in SLE and RA increases activity and expression of hormone-sensitive lipase (HSL) in adipose tissue, which catalyzes the breakdown of TG, releasing free fatty acids (FFA) [12]. Increased FFA entry to liver may elevate hepatic production of VLDL. Relative insulin deficiency also decreases the activity of lipoprotein lipase (LPL), the rate-limiting enzyme of the catabolism of TG-rich lipoproteins [13]. Therefore, reduced LPL activity increases TG and VLDL. HCQ may improve inflammatory state, which normalizes HSL and LPL function and results in improvement of dyslipidemia. However, the underlying mechanisms for HCQ-induced improvement of dyslipidemia should be elucidated in the future.

The present study has limitations. First, the number of studied subjects was small. Second, since this study was retrospective and based on medical charts, lack of data might influence the results. A more detailed prospective study is recommended.

\section{Conclusion}

HCQ was associated with reduction of $\mathrm{HbA1c}$, serum triglyceride, LDL-C and non-HDL-C levels in SLE and RA patients.

\section{Acknowledgments}

We thank the staffs (Yukie Kawamura, Keiko Nakamura, Harue Aoki and Ayano Sakakibara) of the Division of Research Support, National Center for Global Health and Medicine Kohnodai Hospital. 


\section{Financial Disclosure}

Authors have no financial disclosures to report.

\section{Conflict of Interest}

The authors declare that they have no conflict of interest concerning this article.

\section{Informed Consent}

Not applicable.

\section{Author Contributions}

YM and HY designed the research. KH, NT and TK collected data. HY analyzed data, and HY wrote the paper. All authors read and approved the final paper.

\section{References}

1. Belizna C. Hydroxychloroquine as an anti-thrombotic in antiphospholipid syndrome. Autoimmun Rev. 2015;14(4):358-362.

2. Kaplan YC, Ozsarfati J, Nickel C, Koren G. Reproductive outcomes following hydroxychloroquine use for autoimmune diseases: a systematic review and meta-analysis. Br J Clin Pharmacol. 2016;81(5):835-848.

3. Maiorino MI, Bellastella G, Giugliano D, Esposito K. Cooling down inflammation in type 2 diabetes: how strong is the evidence for cardiometabolic benefit? Endocrine. 2017;55(2):360-365.

4. Haque S, Mirjafari H, Bruce IN. Atherosclerosis in rheu- matoid arthritis and systemic lupus erythematosus. Curr Opin Lipidol. 2008;19(4):338-343.

5. Mok CC. Metabolic syndrome and systemic lupus erythematosus: the connection. Expert Rev Clin Immunol. 2019;15(7):765-775.

6. Qiao X, Zhou ZC, Niu R, Su YT, Sun Y, Liu HL, Teng JL, et al. Hydroxychloroquine improves obesity-associated insulin resistance and hepatic steatosis by regulating lipid metabolism. Front Pharmacol. 2019;10:855.

7. Rempenault C, Combe B, Barnetche T, Gaujoux-Viala C, Lukas C, Morel J, Hua C. Metabolic and cardiovascular benefits of hydroxychloroquine in patients with rheumatoid arthritis: a systematic review and meta-analysis. Ann Rheum Dis. 2018;77(1):98-103.

8. Tao CY, Shang J, Chen T, Yu D, Jiang YM, Liu D, Cheng GY, et al. Impact of antimalarial (AM) on serum lipids in systemic lupus erythematosus (SLE) patients: A systematic review and meta-analysis. Medicine (Baltimore). 2019;98(14):e15030.

9. Chen HW, Leonard DA. Chloroquine inhibits cyclization of squalene oxide to lanosterol in mammalian cells. J Biol Chem. 1984;259(13):8156-8162.

10. Sewell RB, Barham SS, LaRusso NF. Effect of chloroquine on the form and function of hepatocyte lysosomes. Morphologic modifications and physiologic alterations related to the biliary excretion of lipids and proteins. Gastroenterology. 1983;85(5):1146-1153.

11. Sachet JC, Borba EF, Bonfa E, Vinagre CG, Silva VM, Maranhao RC. Chloroquine increases low-density lipoprotein removal from plasma in systemic lupus patients. Lupus. 2007;16(4):273-278.

12. Sztalryd C, Kraemer FB. Regulation of hormone-sensitive lipase in streptozotocin-induced diabetic rats. Metabolism. 1995;44(11):1391-1396.

13. Nikkila EA, Huttunen JK, Ehnholm C. Postheparin plasma lipoprotein lipase and hepatic lipase in diabetes mellitus. Relationship to plasma triglyceride metabolism. Diabetes. 1977;26(1):11-21. 